\title{
Enhanced VHD Making Algorithm for 4G Heterogeneous Networks
}

\author{
Sonia', Gurpreet Singh Saini', Chirag Sharma ${ }^{2}$ and Gursharanjeet Singh ${ }^{1}$ \\ 'Department of Electronics and Communication Engineering, Lovely Professional University, Phagwara, \\ Punjab-144411, India; soniabagha4@gmail.com, gurpreet.16889@|pu.co.in, gursharanjeet.singh@lpu.co.in \\ Department of Computer Science and Engineering, Lovely Professional University, Phagwara, Punjab-144411, \\ India; chiragsharma1510@gmail.com
}

\begin{abstract}
In next generation wireless system such as Long Term Evolution (LTE) and Worldwide Interoperability Microwave Access (WIMAX) the major issue is to maintain the quality of service of a network. User required always best connected services anytime and anywhere. Due to increase in number of user on particular network, load is increased on a network. Due to high load handover is required but in many situations unnecessary handoff and handoff failure is initiated, which lags the performance of a network. To overcome this problem we have used RSS vertical handover decision method with some additional parameters to improve the performance in $4 \mathrm{G}$ heterogeneous networks.
\end{abstract}

Keywords: LTE, RSS, Vertical Handoff Decision Algorithms, WIMAX

\section{Introduction}

Wireless communication system generally works through electromagnetic signals those are broadcast by an enabled device within the air or physical environment. The rapid growth of wireless access technologies demands the interoperability and better mobility management techniques to fulfill the requirements of users. In recent years, heterogeneous wireless networks are supposed to create various networks of different types such as WIFI, Worldwide Interoperability for Microwave Access (WIMAX) and Long Term Evolution (LTE) to provide users with promising applications. These networks are independently and vary extensively in terms of their service parameters like throughput, area of coverage, accessing delay etc. The rapid increase of access technologies demands interoperability and better mobility management techniques to fulfill the requirements of users. The big challenge in now days is to keep the mobile station connect anytime and anywhere with best connections ${ }^{1}$. In Recent years mobile terminals are prepared with multiple interfaces and can access a wide range of applications offered by multiple wireless networks. To access the communication services anytime, anywhere with best quality of service and at less cost heterogeneous wireless communication system is a best resolution. In $4 \mathrm{G}$ system such as LTE and WIMAX, handoff execution is more complicated because it not only covers horizontal handoff but also vertical handoffs. Traditionally handoffs were based on RSS (received signal strength) method. In RSS method decision of handover was taken on basis of measurement of received signal 2 . Mobile station measures the signal strength of current network and if the signal strength is getting weaker as the mobile station moving away from base station then it decides to handover the channels to target network. It only handover the call to next selected network if the signal strength of target network is large than the previous network. In case of RSS the signal gets fluctuated due to path loss in wireless medium, due to path loss there is unnecessary handoffs occurs and unnecessary handoffs leads ping pong effect ${ }^{3}$. In this paper to reduce the ping pong effect we proposed vertical handoff decision method with additional parameters such as signal to noise ratio, bandwidth and data rate. This

${ }^{*}$ Author for correspondence 
algorithm provides different set of parameters to provide better handoff. The rest of paper is organized as follows: heterogeneous networks (LTE and WIMAX), first section describes related work, second Section describes the problem arises in traditional method, describes proposed work with additional parameters, next Section discusses the results, discusses the conclusion and last section describes future work.

WIMAX Network: WIMAX is worldwide interoperability microwave access $4 \mathrm{G}$ network. It is a standard for wireless communications used to provide 30-40 megabit per second data rates. The standard for WIMAX is 802.16 which has spectral efficiency of 3.7 (bits per second) per hertz. WIMAX has advantage over WIFI of distance coverage of 30 miles as compare to WIFI covers 300 feet. As we know longer the distance slow down the speed but WIMAX is faster and has more range compared to WIFI. Generally it can provide 1 to 6 miles range with 10 mega bits per second speed. As WIMAX has longer range, faster as compare to WIFI and easily replaceable to DSL, due to this reason communication providers are more excited for WIMAX. WIMAX used at large distance so it offers the broadband connectivity among cities, countries through devices ${ }^{4}$. WIMAX provides low cost network as compare to the third generation. It is now able to provide broadband internet connection access even in remote areas. The WIMAX is replacement of cellular technologies for example GSM and CDMA and it is used to provide more capacity ${ }^{5}$.

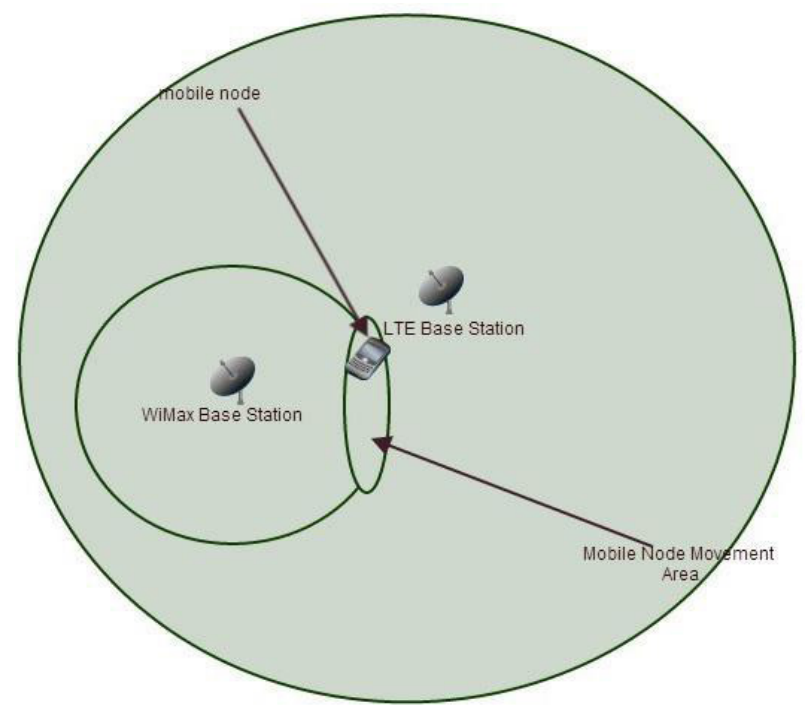

Figure 1. LTE and WIMAX networks ${ }^{5}$
LTE Network: LTE stands for Long Term Evolution or it named as 4G LTE technology. This technology based on GSM and UMTS technologies. LTE is a standard for wireless communication technologies and it provides high speed of data, increased capacity and high speed for different radio interface networks. The main objective of this LTE technology is to enhance the capacity, increased the speed of wireless data networks by using modulation schemes and digital networks by using modulation schemes and digital signal processing techniques ${ }^{5}$. LTE is used to operate on different radio spectrum because it is incompatible with $2 \mathrm{G}$ and $3 \mathrm{G}$. LTE technology offers uplink rate 75 Mega bits per second and downlink rates of 299 Mega bits per second. This technology can handle fast moving mobiles and can support multi cast and broadcast services ${ }^{\underline{6}}$ (Figure 1).

\section{Related Work}

Zahran and Liang ${ }^{7}$ proposed RSS based VHD algorithm using lifetime metric which decreasing number of superfluous handoffs and enhanced average throughput for the users. Its drawback is high packet delay.

Mohanty and Akyildiz ${ }^{-}$proposed RSS based VHD algorithm comparing the traditional RSS and a dynamic RSS threshold. This method reduced the false handover triggering and handover failure probabilities. Its drawback is wastage of network resources.

Yan et al.. developed an RSS based VHD algorithm that takes into account the time the mobile terminal is expected to spend within a WLAN cell. This method reduces handover failures, unnecessary handovers and connection breakdowns. Its drawback is large handover delay.

Lee et al..$^{10}$ proposed a bandwidth based VHD algorithm which give more system throughput and low handover delay for real time applications. Its drawback is difficulty in acquiring available bandwidth information and increased new application blocking rate.

Yang et al. $\frac{12}{}$ provide a bandwidth based VHD algorithm using Signal to Interference and Noise Ratio (SINR).Its draw-back is unnecessary handovers.

$\mathrm{In}^{12}$ Chi et al. present a VHD heuristic based on the Wrong Decision Probability (WDP) prediction. This algorithm minimized unnecessary handover probability and balanced the traffic load. Its drawback is increased 
connection breakdown probability without considering the RSS.

\section{Problem Description}

The traditional method used RSS based algorithm in which the Received Signal Strength (RSS) measurement is used to take decision for selection of appropriate target network ${ }^{13}$. In RSS based algorithm handover is considered based on calculated received signal from nearest base station. When a mobile station is present in one network it continuously receives good quality signal from nearest base station but when mobile station is moving away from base station the signal quality gets weaker. Before the signal gets totally weak we need to handover the call to another network. The mobile station continuously measures the received signal strength using moving average method:

\subsection{RSS Current Network > RSS Old Network}

RSS based algorithm is useful for network selection criteria. But it is more suitable for homogeneous networks ${ }^{14}$. In RSS based algorithm mobile station calculates the signal strength from base station and then take decision. But due to wireless medium there are fluctuations in signal due to path loss, this is known as fading. Due to large variation in signal unnecessary handoffs are initiated which degrades the performance of network. In RSS based algorithm handoff is occur when received signal quality is low but we know that received signal is a random signal due to wireless medium the signal may get down and may enhance suddenly i.e. the signal is fluctuated signal and this may result in early and late handoffs which is called as ping pong effect. We cannot take decision based on RSS only as it will be wastage of resources. So for better handover management only RSS based vertical algorithm is not sufficient. We need to use some more parameters to measure the network conditions.

\section{Proposed Vertical Handoff Decision Algorithm}

In future generation of wireless environment every user's requirement is seamless mobility and continuation access of services with best quality anytime and anywhere $e^{\underline{15}}$. In above section we have discussed RSS based algorithm in which handover decision was taken on basis of received signal from base station. RSS based handover decision is not enough for appropriate handover as there may problem of early and late handoff. So to enhance the performance of network, we have introduced proposed vertical handoff decision algorithm with additional parameters such as bandwidth, throughput data rate $e^{16}$. In this paper we have considered two networks LTE and WIMAX and handoff will take place on basis of additional parameters added to RSS. In this scenario when a mobile terminal positioned partially between two networks LTE and WIMAX, according to traditional algorithm it calculate RSS of both networks, if RSS of WIMAX is less than the RSS calculated of LTE network then it will decide to handover to LTE. In proposed RSS based vertical handover decision algorithm, we will also calculate bandwidth, throughput and data rate of two networks to handover. In proposed algorithm when a mobile terminal is moving from LTE network toward WIMAX network and at the boundary of WIMAX network mobile terminal will detect two signals simultaneously in which one is of LTE and other signal is of WIMAX network. At this point mobile will receive low signal quality of LTE network and high signal quality of WIMAX network but according to proposed method we will also calculate additional parameters such as data rate, bandwidth and throughput of both networks and based on these all parameters handover will be taken into account. This proposed method is clearly illustrated in flow chart Figure 2.

For Received signal measurement we need to measure the path loss of signal occurs due to reflection, diffraction of signal in wireless medium ${ }^{14}$. So path loss is calculated as:

$\operatorname{Pr} \mathrm{dBm}=\mathrm{Pt} \mathrm{DBm}+K \mathrm{~dB}-10 \log 10[\mathrm{~d} / \mathrm{do}](3.3)$

In this equation $\mathrm{Pr}$ is received power, $\mathrm{Pt}$ is transmitted power, $K$ is a unit less constant which depends on the antenna characteristics and the average channel attenuation, do is a reference distance for the antenna far-field, and is the path loss exponent. Transmission distances $\mathrm{d}$ $>$ do, where do is typically assumed to be 1-10 $\mathrm{m}$ indoors and 10-100 m outdoors.

The received power is defined as the transmitted power and the path loss, shadowing, and multipath fading. The noise power is described by the bandwidth of the transmitted signal and the spectral properties of $n(t)$. Specifically, if the bandwidth of the complex envelope $u$ 
(t) of $s(t)$ is $B$ then the bandwidth of the transmitted signal $s(t)$ is $2 B$. Since the noise $n(t)$ has uniform power spectral density $\mathrm{No} / 2$, the total noise power within the bandwidth $2 B$ is:

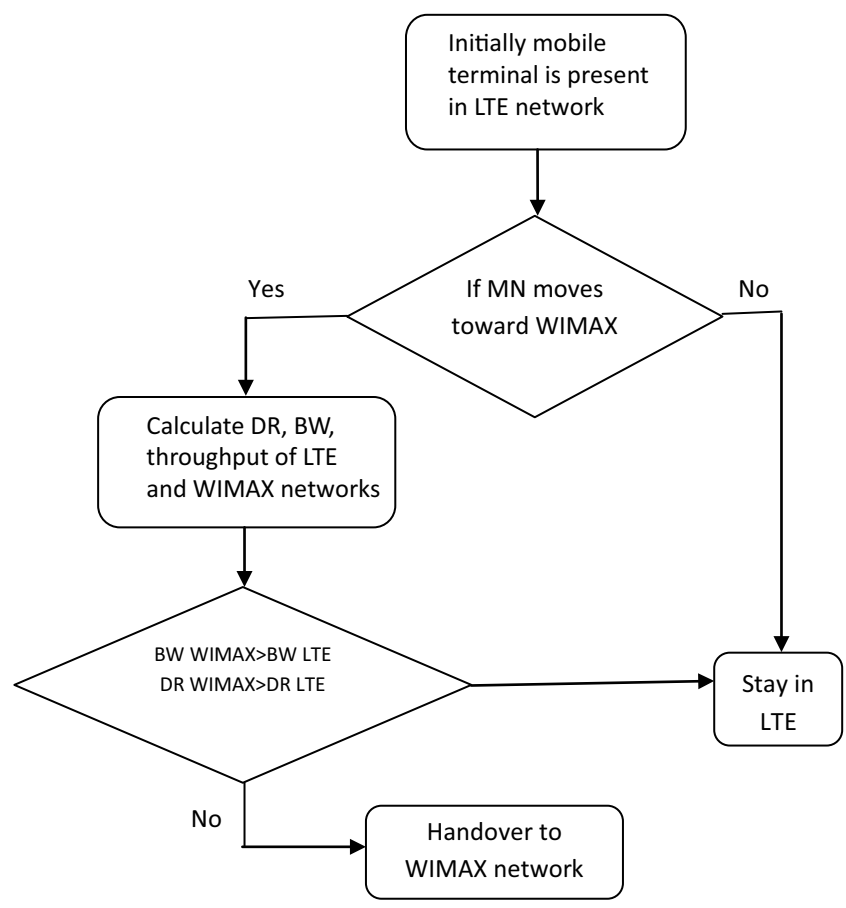

Figure 2. Flow chart of proposed VHD algorithm

$\mathrm{N}=\mathrm{No} / 2^{\star} 2 \mathrm{~B}=\mathrm{No} \mathrm{B}$

Now the received signal to noise ratio is calculated as signal power to noise power ${ }^{17}$.

$\mathrm{SNR}=\mathrm{Pr} / \mathrm{No}^{\star} \mathrm{B}$

The Received Signal for two networks is measured individually. Throughput is measured in bits per second. Throughput is the sum of the data rates that are delivered to all terminals in a network.

Throughput $=$ sum (data rate)

Data rate is the basic characteristics of communication system and it is defined as the rate at which bits are transmitted over the medium in bits/ sec or bps we can derive the data rate by multiplying mutual information with bandwidth of channel as mentioned below:

Data rate $=\mathrm{MI} \times \mathrm{BW}$

In above flow chart, When a mobile network moving from LTE network towards WIMAX network area, the mobile network always measures the signal and at the boundary of LTE network it will compare the RSS received from both networks and take decision of handover but in proposed scheme decision time mobile network calculates data rate, bandwidth and throughput as well and then selects the better performance network to handover ${ }^{16}$. Mobile network will not be depend on RSS calculations, it will only compare another additional parameters to improve the performance of network so that there should not be early or late handoffs ${ }^{17}$.

\section{Results Analysis}

We have analyzed the two heterogeneous $4 \mathrm{G}$ networks LTE and WIMAX. For Simulation the TOOL used is MATLAB. For simulation we have created LTE and WIMAX network. WIMAX is a microcell in LTE network area. WIMAX has 600 coordinates and LTE has 10 coordinates. We have Simulate the two networks and achieved best quality of service with simulation parameters shown in Table 1.

Table 1. Simulation parameters

\begin{tabular}{|l|l|}
\hline Parameters Used & Values \\
\hline WIMAX Coordinates & 600 \\
\hline LTE Coordinates & 10 \\
\hline LTE Frequency & $2000^{\star} 10^{\wedge} 6 \mathrm{~Hz}$ \\
\hline WIMAX Frequency & $2500^{\star} 10^{\wedge} 6 \mathrm{~Hz}$ \\
\hline Bandwidth range for LTE & $4-20 \mathrm{MHz}$ \\
\hline Bandwidth range for & $2-10 \mathrm{MHz}$ \\
\hline WIMAX & \\
\hline Mobile station speed & $40 \mathrm{~m} / \mathrm{s}$ \\
\hline
\end{tabular}

By using these simulation parameters we have analyzed the performance of two networks with additional parameters such as bandwidth, data rate and throughput for LTE and WIMAX both when the mobile terminal is moving toward WIMAX network. In Figure 3, we have analyzed the throughput of two networks at the time of movement of node and we have observed the throughput of LTE network is more than throughput of WIMAX network. As LTE is a wide network and having more through put so at the boundary of WIMAX mobile network will detect high performance of throughput of LTE network than WIMAX network.

Figure 4 describes the Bandwidth of LTE and WIMAX network using additional parameters with RSS handover algorithm. We have analyzed that bandwidth provided for LTE network is higher than WIMAX network. As 
distance is increasing of mobile node still LTE has more bandwidth than WIMAX network. So this result shows that LTE network is best network for mobile nodes with movement at any speed and distance.

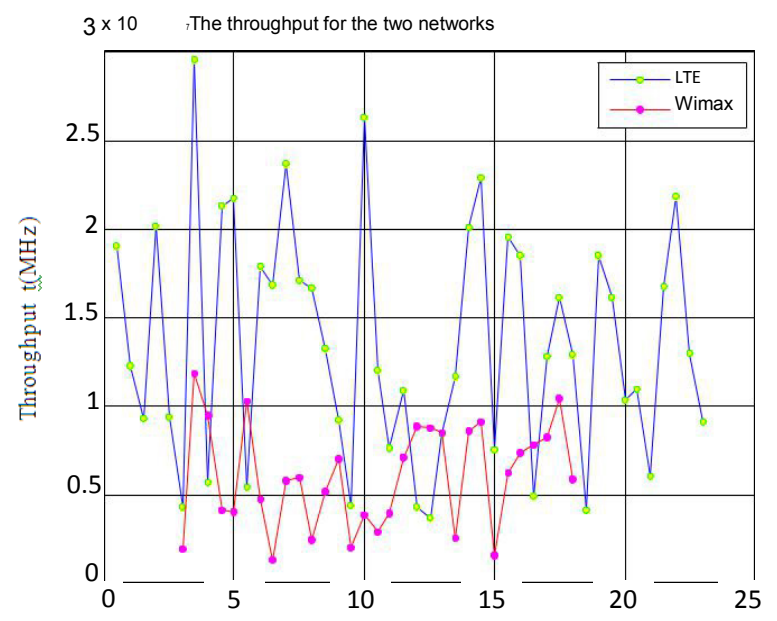

Figure 3. Throughput of two networks

$2 \times 10$ The band width for the two networks

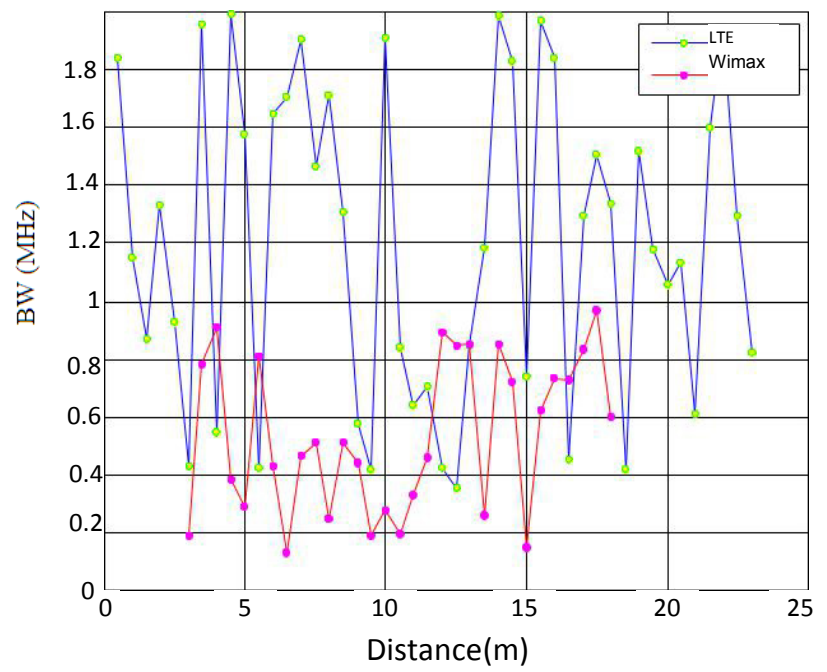

Figure 4. Bandwidth for LTE and WIMAX network

Figure 5 illustrates the data rate of two networks. From analysis we have observed that there are variations in calculated data rate but on average the data rate of LTE is higher than WIMAX network. As the distance is increased WIMAX network data rate have very small value as compare to LTE network.

Figure 6 describes the state of mobile terminal and shows that the state of mobile terminal having high data rate when handover occurs. This result shows that during handover the user cannot face low signal quality. Using additional parameters data rate of mobile network have higher performance.

$3 \times 10$ The data rate for the two networks

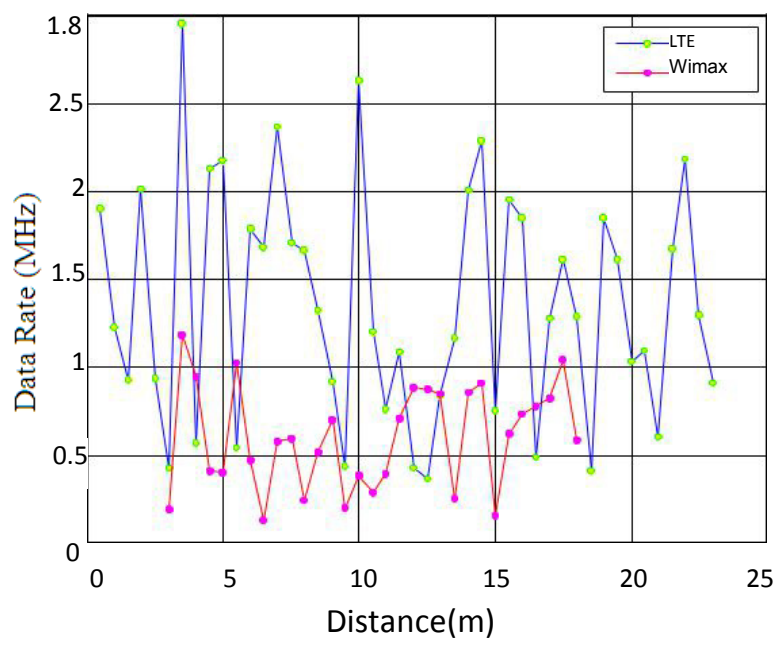

Figure 5. Data rate for LTE and WIMAX network

$3 \times 10 \quad$ The data rate for the mobile station

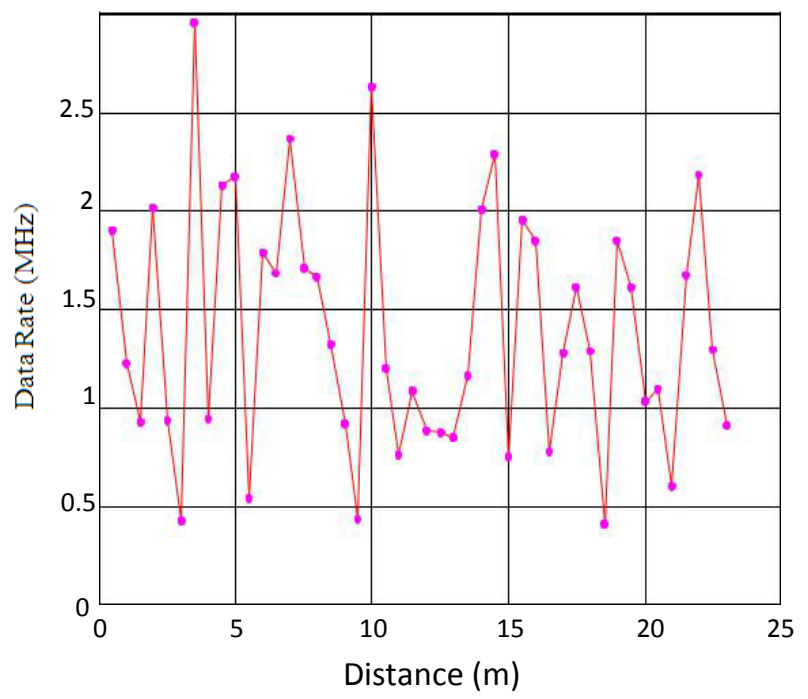

Figure 6. Data rate for mobile station

Figure 7 describes the throughput of mobile network at the time of handover. We have analyzed the performance of mobile network while switching from one network to another and Using proposed method throughput of mobile network has good performance while handover the channels. The main goal we have achieved that the best signal quality at any distance and at any speed of moving mobile nodes. 
$5 \times 10$ The throughput for the mobile station

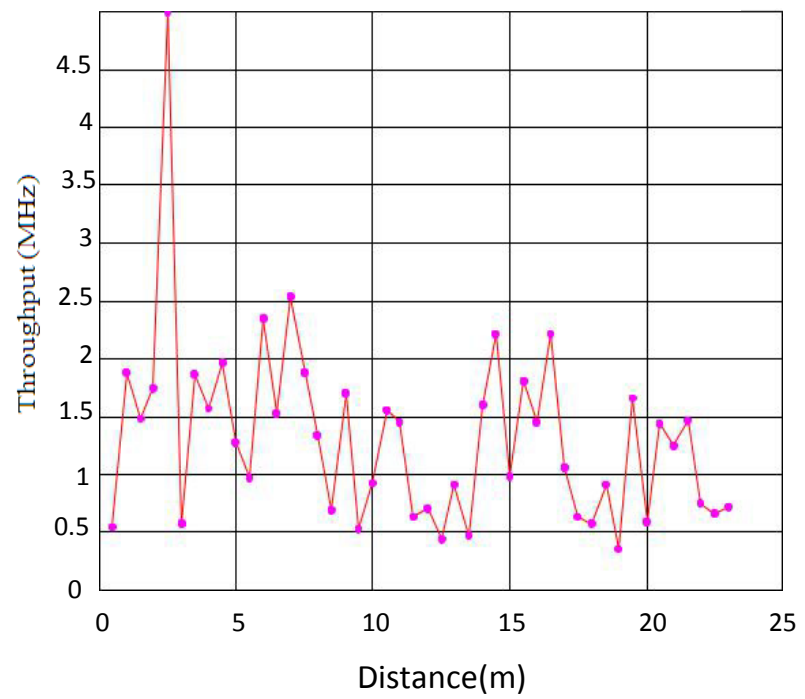

Figure 7. Throughput of mobile station

\section{Conclusion}

We know that today's world is $4 \mathrm{G}$ world, everyone want to use best quality of service. But in $4 \mathrm{G}$ wireless networks due to high mobility of nodes best quality of service is still a challenging task. User wants to access high speed and best services at anytime and anywhere. The performance degrades due to high mobility of vehicles speed. In this era the best quality of service cannot achieved only with RSS based decision algorithm, due to fluctuations in received signal from base station we cannot achieve the desired output, so to obtain best performance in $4 \mathrm{G}$ heterogeneous networks proposed handoff algorithm is used with some additional parameters with RSS scheme. In proposed algorithm we are using bandwidth, data rate and throughput with RSS scheme to enhance the performance. By using proposed method we have achieved better performance of vertical handover with SNR and bandwidth as compare to previous method.

\section{Future Work}

In $4 \mathrm{G}$ heterogeneous networks better quality of service is achieved with proposed algorithm of vertical handover in LTE and WIMAX networks. In future of this research we can improve the work in direction to test and analyze the proposed algorithm with additional parameters using complex heterogeneous networks. The proposed algorithm is used to reduce ping pong effect and enhance the performance of network. In future we can improve the performance by enhancing this approach or by analyze this approach with other complex parameters of network and we can further improve the probability of call dropping and packet loss ratio.

\section{References}

1. Bhuvaneswari A. An Overview of Vertical Handoff Decision Making Algorithms, Journal of Computer Network and Information Technology. 2012; 9.

2. Brahmjit Singh. An Improved Handover Algorithm Based on Signal Strength Plus Distance for Interoperability in Mobile Cellular Networks, Wireless Pers. Commun. 2007; 43:879-87.

3. Ken Ichi Itoh. Performance of Handoff Algorithm Based on Distance and RSSI Measurements, IEEE Trans. Veh. Tech. 2002; 51:1460-68.

4. Issaka Hassane Abdoulaizi, Li Renfa, Zeng Fanzi. Handover Necessity Estimation for $4 \mathrm{G}$ Heterogeneous Networks, International Journal of Information Science and Techniques. 2012; 2(1).

5. Smita Ramsey, Ravi Shankar Shukla, Zubair Khan. RSS Based Adaptive Hysteresis Vertical Handoff Decision Algorithm between WIMAX and LTE to Reduce Ping-Pong Effect, International Journal of Scientific and Engineering Research. 2013; 8(4).

6. Mohammed Nour Hindia, Ahmed wasif Reza. Enhancement the Handovers Accuracy and Performance of WIMAX and LTE Networks, International Conference on Convergence Information Technology. 2014; 5.

7. Zahran AH, Liang B, Saleh A. Signal Threshold Adaptation for Vertical Handoff in Heterogeneous Wireless Networks, Mobile Networks and Applications. 2006.

8. Mohanty S, Akyildiz IF. A Cross-Layer (layer 2+3) Handoff Management Protocol for Next-Generation Wireless Systems, IEEE Transactions on Mobile Computing. 2006.

9. Yan X, Mani N, Ahmet Sekercioglu Y. A Traveling Distance Prediction Based Method to Minimize Unnecessary Handovers from Cellular Networks to WLANs, IEEE Communications Letters. 2008.

10. Ken Ichi Itoh. Performance of Handoff Algorithm Based on Distance and RSSI Measurements, IEEE Trans. Veh. Tech. 2002; 51:1460-68.

11. Issaka Hassane Abdoulaizi, Li Renfa and Zeng Fanzi,2012,"Handover Necessity estimation for 4G Heterogeneous Networks", International journal of information science and techniques, volume 2,No.1.

12. Smita Ramsey, Mr. Ravi Shankar Shukla, Mr. Zubair Khan,2013," RSS Based Adaptive Hysteresis Vertical Handoff Decision Algorithmbetween WIMAX and LTE 
to Reduce Ping-Pong Effect", International Journal of Scientific \& Engineering Research, Issue 8, volume 4.

13. Mohammed Nour Hindia ,Ahmed was if Reza,2014," Enhancement the handovers accuracy and performance of WIMAX and LTE networks", International Conference on Convergence Information Technology,vol. 5.

14. A.H. Zahran, B. Liang, A. Saleh,2006, "Signal threshold adaptation for vertical handoff in heterogeneous wireless networks", Mobile Networks and Applications.

15. Dang Duc Nguyen, Yang Xia, Mai Ngoc Son. A Mobility Management Scheme with QoS Support for Heterogeneous Multimode Mobile Nodes, IEEE. 2008; 8.

16. Xiaohuan Yan, Ahmet Sekercioglu Y, Sathya Narayanan. A Survey of Vertical Handover Decision Algorithms in Fourth Generation Heterogeneous Wireless Networks, Elsevier Journal. 2010.

17. Kemeng Yang, Iqbal Gondal, Bin Qiu, Laurence S. Dooley. Combined SINR Based Vertical Handoff Algorithm for
Next Generation Heterogeneous Wireless Networks, Global Telecommunications Conference: Washington DC, 2007.

18. Karl Anderson, Daniel Granlund, Muslim Elkotob, Christer Ahlund,2010, "Bandwidth Efficient Mobility management for heterogeneous wireless networks"IEEE communication society, volume 10 .

19. N. P. Singh and Brahmjit Singh, 2011," Improved vertical handover decision algorithm for UMTS-WLAN," International Journal of Future Generation Communication and Networking, vol. 4, issue 4, pp. 113-123.

20. Richa, Shelej Khera, 2014, " QoS parameter based vertical handoff decision in Heterogeneous Networks", International Journal of Advanced Research in computer and communication Engineering, Volume 3.

21. Hashem, 2009,'A fuzzy logic based adaptive handoff management protocol for next generation wireless system", IEEE Journal of Networks, volume 4. 